\title{
Gentle method for removing metal and restoring function after scanning electron microscopy
}

\author{
Rashi Sharma, ${ }^{a}$ Jennefir L. Digaum $\odot{ }^{b}$ Hannah West, ${ }^{b}$ Casey M. Schwarz, \\ and Stephen M. Kuebler $\odot^{\text {a,b,d, }}$, \\ ${ }^{a}$ University of Central Florida, Department of Chemistry, Orlando, Florida, United States \\ ${ }^{b}$ University of Central Florida, CREOL, The College of Optics and Photonics, Orlando, \\ Florida, United States \\ ${ }^{c}$ Ursinus College, Department of Physics and Astronomy, Collegeville, Pennsylvania, \\ United States \\ ${ }^{\mathrm{d}}$ University of Central Florida, Department of Materials Science and Engineering, Orlando, \\ Florida, United States
}

\begin{abstract}
An etching process is demonstrated for removing noble metal from microstructures to restore their original function after being characterized by scanning electron microscopy (SEM). Using neither aggressive acids nor high temperatures, the etching method gently removes gold/ palladium alloys from complex three-dimensional microstructures, preserving their structural form. To explore the efficacy of the etching process, polymeric photonic crystals and monolithic microstructures were fabricated, metal coated, etched, and then structurally and optically characterized. Metal coating substantially diminishes the optical functional and transmission of the microstructures. SEM imaging performed throughout a series of metal sputtering, etching, and resputtering shows that the etching process does not significantly alter the form of a microstructure. Measurements of optical transmission using a scanned-optical-fiber system confirm that the etchant removes the metal and restores the optical properties of the microstructures. (C) The Authors. Published by SPIE under a Creative Commons Attribution 4.0 Unported License. Distribution or reproduction of this work in whole or in part requires full attribution of the original publication, including its DOI. [DOI: 10.1117/1.JMM.20.2.023601]
\end{abstract}

Keywords: etching; scanning electron microscopy; microfabrication; photonic crystals.

Paper 21020 received Mar. 15, 2021; accepted for publication Apr. 22, 2021; published online May 6, 2021.

\section{Introduction}

Micro- and nanoscale three-dimensional (3D) structures are important for a wide range of applications including photonics, ${ }^{1,2}$ micro-electrical mechanical systems (MEMS), ${ }^{3}$ micro-optoelectro-mechanical system (MOEMS), ${ }^{4}$ and bioscaffolds. ${ }^{5,6}$ Some widely used techniques for fabricating 3D structures include multi-photon lithography (MPL), ${ }^{7}$ e-beam lithography, ${ }^{8}$ and multi-step mask-based lithography. ${ }^{9}$ 3D forms can be structurally characterized by confocal microscopy, ${ }^{10} \mathrm{x}$-ray tomography, ${ }^{11}$ and multi-photon imaging, ${ }^{12}$ but scanning electron microscopy (SEM) is most commonly used, given its superior resolution and versatility. SEM is best suited for imaging conductive samples, because negative charge that accumulates on the sample deflects incident electrons and reduces the resolution. Functional microstructures can be created with biomaterials, ${ }^{13}$ ceramics,${ }^{14}$ polymers,${ }^{15}$ glasses,${ }^{16}$ and semiconductors. ${ }^{17}$ Structures made from these materials are typically sputtered with metal prior to SEM imaging because they are poor electrical conductors. Non-conducting samples can be imaged using variable-pressure SEM (VPSEM), but the resolution is poor compared to that which can achieved by high-vacuum SEM imaging of sputter-coated samples. ${ }^{18}$ High-vacuum SEM is also much more widely available than VPSEM.

Sputtering is used to coat a structure under low vacuum with a thin layer of metal ( $20 \mathrm{~nm})$, typically gold $(\mathrm{Au})$, gold/palladium $(\mathrm{Au} / \mathrm{Pd})$, platinum, chromium, or silver. $\mathrm{Au}$ and $\mathrm{Au} / \mathrm{Pd}$ are

*Address all correspondence to Stephen M. Kuebler, kuebler@ucf.edu 
most commonly used for SEM imaging because they produce a small metal grain size that minimally distorts apparent dimensions of the structure. Sputter coating a sample with metal also helps to protect it from thermal damage from the electron beam during SEM imaging, which is particularly useful for soft materials such as polymers. Sputter-coating 3D microstructures with metal radically change their physical properties, making them unsuitable for subsequent optical, electrical, or other characterization. Although the structural form of a sample is preserved during SEM imaging, the technique is essential destructive when it must be preceded by metal sputtering.

Methods have been reported for removing metal coatings from samples, but each of these suffers from one or more limitations. Miller et al. ${ }^{19}$ used the output from a Nd:YAG laser to ablate $\mathrm{Au} / \mathrm{Pd}$ from surfaces. This approach involves minimal sample handling, which reduces risk of damaging the sample, but it is expensive, time-consuming, and could potentially damage an underlying sample. Cyanide has been used to remove $\mathrm{Au}$ or $\mathrm{Au} / \mathrm{Pd}$ chemically from archeological samples after SEM imaging. ${ }^{20-23}$ These approaches are inexpensive, fast, and non-damaging to the sample, but cyanide is highly toxic. In 2012 Jones et al. ${ }^{24}$ reported a non-destructive and safe method for etching Au from archeological specimens using iodine $\left(\mathrm{I}_{2}\right)$ as an oxidizing agent, dissolved in an ionic liquid. To the best of the authors' knowledge, this method has not been applied to artificial microstructures, nor has it been used to remove $\mathrm{Au} / \mathrm{Pd}$ or demonstrate the restoration of structural function after SEM imaging.

This work reports a modified application of the method of Jones et al. and demonstrates how it can be used to remove sputtered $\mathrm{Au} / \mathrm{Pd}$ from 3D microstructures after SEM imaging to restore both form and function. Changes in handling the etchant were introduced to make the process gentler toward the microstructures. The structural and optical behaviors of etched samples were investigated by comparing SEM images and measurements of optical function before and after etching. To the best of the authors' knowledge, this is the first-time etching Au/Pd-coatings from polymeric 3D microstructures have been studied to determine if form and function can be restored after sputter coating. The data from this study show that the etching process can be used to remove sputtered metal and restore a microstructure's form and function.

\section{Experimental}

\subsection{Fabrication and Structural Characterization}

Polymeric 3D microstructures and devices were fabricated by MPL in IP-Dip, a commercially available cross-linkable acrylate photopolymer (NanoScribe), using methods described previously. ${ }^{25}$ In preparation for SEM imaging, the 3D microstructures were sputter coated (Emitech K675X) with a 20-nm thick layer of Au/Pd. A Zeiss Ultra 55 FEG SEM was used to image metal-coated 3D microstructures before and after etching.

\subsection{Preparation of Etching Solutions}

Ethaline solutions were prepared ${ }^{24}$ by mixing choline chloride (Sigma-Aldrich $\geq 98 \%$, CAS 67 48-1) and ethylene glycol (Sigma-Aldrich $\geq 98 \%$, CAS 107-21-1) in a 1:2 molar ratio and heating to $60^{\circ} \mathrm{C}$ for $5 \mathrm{~min}$ to $10 \mathrm{~min}$ to dissolve all solids completely. Ethaline can be prepared in advance and kept in a sealed plastic or glass container under cool and dry conditions for future use. With time, choline chloride may crystallize and settle at the bottom of the container, but it can be redissolved by heating the solution immediately prior to use.

Etchant solutions were prepared immediately prior to use by dissolving $\mathrm{I}_{2}$ (Fisher Scientific $\geq 99.5 \%$, CAS 7553-56-2) in ethaline to a concentration of $0.125 \mathrm{M}$ at $60^{\circ} \mathrm{C}$. Saturated aqueous potassium iodide (KI) was prepared by dissolving $150 \mathrm{~g}$ of KI (Acros Organics, CAS 7681-11-0) in $100 \mathrm{~mL}$ of deionized water $(18 \mathrm{M} \Omega)$ with constant stirring at $20^{\circ} \mathrm{C}$.

\subsection{Metal Etching}

A metal-coated microstructure was placed in a $10-\mathrm{mL}$ glass beaker. Warm $\mathrm{I}_{2}$-etchant $(2 \mathrm{~mL}$, $60^{\circ} \mathrm{C}$ ) was gently added dropwise to avoid dislodging the structure from the substrate. The 
submerged sample was maintained at $60^{\circ} \mathrm{C}$ for $15 \mathrm{~min}$. The etchant was then removed slowly using a pipette, and the sample was rinsed by soaking in saturated KI (15 min, two portions), followed by deionized water $(10 \mathrm{~min})$. The water was then removed by pipette, and the sample was dried in air.

\subsection{Optical Characterization of Structures}

The optical properties of 3D microstructures were measured using the scanned-optical-fiber system shown in Fig. 1(a). An early version of the system is reported elsewhere,${ }^{26}$ and additional enhancements are described here. Light injected into the scanned-fiber system had a wavelength in vacuum of $\lambda_{0}=1.55 \mu \mathrm{m}$ and was derived from an optical parametric amplifier pumped by an amplified femtosecond laser (coherent). The laser beam was routed through an optical filter to remove residual components having $\lambda_{0}<1 \mu \mathrm{m}$ and then split into three "source" beams: (1) a main beam, used for optical alignment and calibration; (2) a zero-order diffracted beam, used for broad-bandwidth optical characterization; and (3) a spectrally dispersed, first-order diffracted beam was used for narrowband optical characterization. The bandwidth of the source beams was measured using a spectrophotometer (OceanOptics NIRQuest512-2.2 NIR). The beams

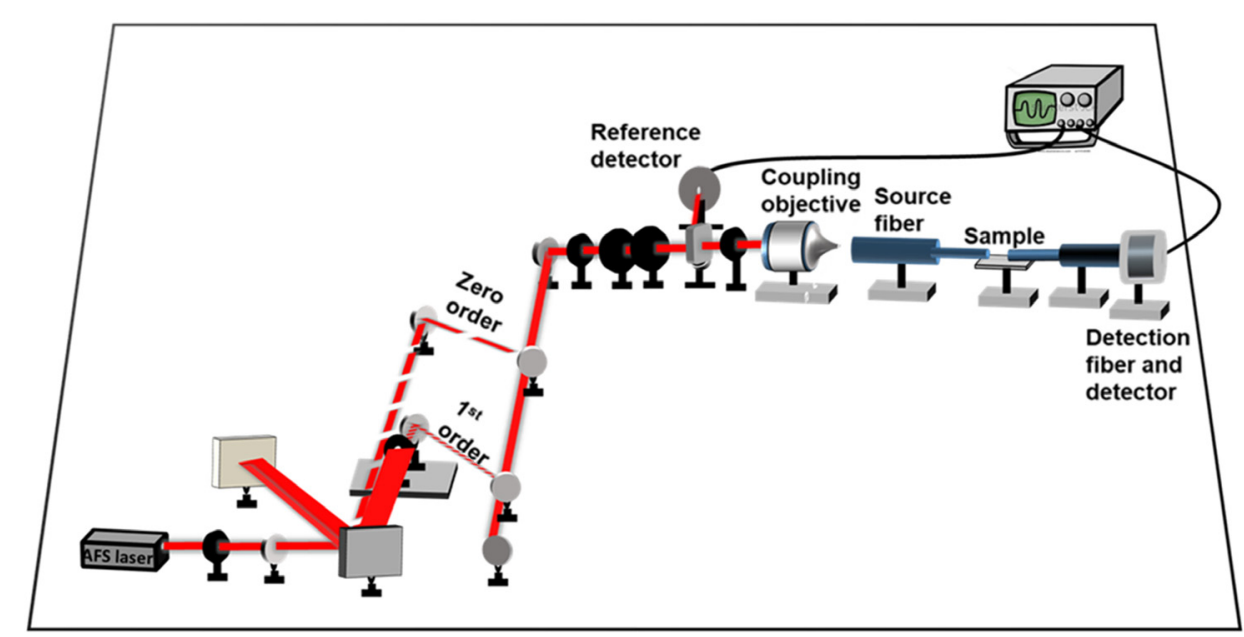

(a)

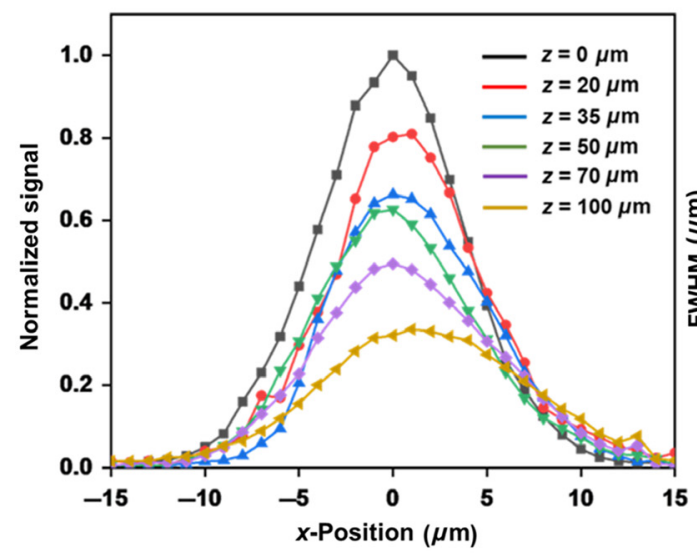

(b)

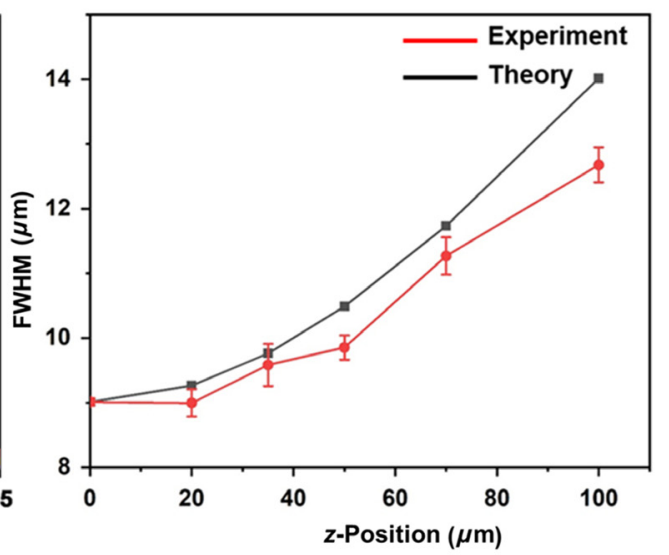

(c)

Fig. 1 (a) Scanned-optical-fiber system used to characterize optical beams transmitted through the microstructures. (b) Line scans illustrating natural divergence of light exiting the source fiber and propagating distance $z$. (c) Comparison of theoretical and experimentally measured widths of line scans (as FWHM) for light exiting the source fiber. The theoretical scan widths were obtained by propagating a Gaussian beam exiting the source fiber and convoluting its width at position $z$ with the response function of the detection fiber. 
were combined onto a common path, but optical measurements were performed using only one source beam at any given time. An objective lens coupled the source beam into a single-mode "source fiber" (Thorlabs 1550BHP, mode-field diameter $=9.5 \mu \mathrm{m} \pm 0.5 \mu \mathrm{m}$ ) that delivered light onto a microstructure under study. A "detection fiber" of the same type on the opposite side of the sample was coupled to a PbSe detector (ThorLabs PDA20H) and used to record beam profiles, measure optical transmission, and estimate the spot size of an emerging beam. A computer-controlled three-axis stage scanned the output fiber spatially during automated measurements. Signals were normalized against that from a reference detector to remove shot-to-shot fluctuations due to the laser.

The width of a beam emerging from the source fiber, and/or transmitted through a microstructure, was obtained by recording signal $S(x, y, z)$ as the detection-fiber scanned across the beam in the $x y$ planes, and at varying distance $z$ between the source and detection fibers. Figure 1(b) shows a series of line scans obtained by measuring signal for the beam directly exiting the source fiber. All line scans have a Gaussian shape which increases with $z$. The signal $S$ is a convolution of the irradiance profile of the beam with the input response function of the detection fiber. A single-mode, step-index fiber outputs a beam that can be approximated as having a Gaussian profile of the form in Eq. (1) ${ }^{27,28}$ The input response function of the detection fiber can also be assumed to be Gaussian, and their convolution should then yield a Gaussian signal profile that widens with $z$, as seen in Fig. 1(b) ${ }^{29}$

Line scans like those in Fig. 1(b) were fit to a Gaussian function to obtain the $1 / e^{2}$-width $=$ FWHM $/(2 \ln 2)^{1 / 2}$, where FWHM denotes the full-width at half-maximum. Sputter-coated devices only partly transmitted incident light and generated distorted beams that could not be meaningfully fit to a Gaussian function, so to facilitate comparison, all line scans presented in Sec. 3 are compared in terms of FWHM. But here the performance of the scanned-fiber system and the ability to detect changes in beam size can be demonstrated by comparing experimentally measured scan widths to those predicted for a Gaussian beam exiting the source fiber and diverging in air. ${ }^{30}$ When the source and detection fibers are coupled end-to-end $(z=0)$, the measured signal $S$ is a convolution of the input response function of the fiber with itself, so scan width $(0)=w_{0} \sqrt{ } 2$, where $w_{0}$ is the $1 / e^{2}$-width of the beam at $z=0$. Figures $1(\mathrm{~b})$ and 1 (c) show that the line scan $S(z=0)$ has FWHM $=9.0 \mu \mathrm{m}$, which gives $w_{0}=5.4 \mu \mathrm{m}$. The corresponding mode-field-diameter is $2 w_{0}=10.4 \mu \mathrm{m}$, which agrees with the value provided by the manufacturer of the fiber. Upon propagating distance $z$, the beam diverges to $w(z)$ calculated with Eqs. (2) and (3). ${ }^{31}$ Convolution of the diverged beam with the input response function of the fiber gives theoretical scan width $(z)=\left[w(z)^{2}+w_{0}^{2}\right]^{1 / 2}$. The theoretical and experimentally measured scan widths for the beam exiting the source fiber are plotted together in Fig. 1(c) and seen to agree well. This example illustrates that changes in the beam width of a few microns can readily be observed using the scanned-fiber system:

$$
\begin{gathered}
I(r, z)=I_{0} \exp \left[-2 r^{2} / w(z)^{2}\right], \\
w(z)=w_{0} \sqrt{1+\left[\frac{z}{z_{R\left(w_{0}\right)}}\right]^{2}}, \\
z_{R}\left(w_{0}\right)=\frac{\pi}{\lambda_{0}} w_{0}^{2} .
\end{gathered}
$$

Microstructures were optically characterized by inserting them between the source and detection fibers and performing line scans across the beam transmitted through the sample. Changes in the width of the scans were used to observe how the structure altered propagation of the beam, and how that varied between pristine, metal-coated, and etched samples. Optical transmission through the sample was obtained as the ratio of the peak signal before and after the structure. Ratios of the integrated incident and transmitted signals confirmed that the peak signals provide a suitable measure of optical transmission. Uncertainties were obtained by comparing the variation between repeat measurements with the standard error obtained from fits and reporting the larger of the two. 


\section{Results and Discussion}

\subsection{Processing Samples with Etchant}

The etchant solution is dark purple due to dissolved $\mathrm{I}_{2}$, so the removal of metal from the sample is not easily observed during etching. Because the etching process is mild, no bubbling, foaming, or other obvious signs of chemical reaction are visible. After the etch time has elapsed, the sample is removed, rinsed with saturated aqueous KI, then rinsed with water. Rinsing the sample first with pure water would precipitate microcrystals of $\mathrm{I}_{2}$ onto the surface and within internal voids of the microstructure. Therefore, the sample is rinsed first with saturated KI because $\mathrm{I}^{-}$ reacts with $\mathrm{I}_{2}$ forming soluble $\mathrm{I}_{3}{ }^{-}$, which is soluble in water.

After rinsing, the effect of the etching is immediately obvious from a change in reflectivity of the sample, as seen in Fig. 2. Au/Pd sputter coated onto the sample for SEM imaging makes the supporting substrate reflective, to a degree that depends upon the thickness of the metal layer, but the camera used to record the images is clearly visible [Fig. 2(a)]. After etching, the substrate is again highly transmissive [Fig. 2(b)], indicating that metal was removed.

Generally, strong oxidizing agents are needed to etch $\mathrm{Au}, \mathrm{Pd}$, and other noble metals. Yet strong oxidizers can destroy soft materials like polymers, fragile microstructures, and adhesion layers that bind a structure to its substrate. $\mathrm{I}_{2}$ is a mild oxidizing agent that does not attack saturated polymers, and $\mathrm{I}_{2}$ by itself is not strong enough to oxidize noble metals. The species $\mathrm{Au}^{3+}, \mathrm{Pd}^{2+}$, and oxidized forms of related noble metals form stable complexes with chloride anions, so the base metals are easier to oxidize in the presence of $\mathrm{Cl}^{-} .32,33$ The etching agent used in this work is based on ethaline, a eutectic mixture of choline chloride, and ethylene glycol, which readily dissolves $\mathrm{I}_{2}$ and provides a high concentration of $\mathrm{Cl}^{-}(\sim 5 \mathrm{M})$. In ethaline, $\mathrm{I}_{2}$ is strong enough to oxidize $\mathrm{Au}$ and $\mathrm{Pd}$, so the etchant can dissolve the noble metals without harming the polymeric microstructures.

\subsection{Structural and Optical Characterization of Etched Photonic Crystals}

Uniform-lattice photonic crystals (ULPCs) that self-collimate light ${ }^{34}$ were fabricated and characterized to study how the etching process affects the optical properties of a nanophotonic device. The self-collimation effect suppresses beam divergence, so a light beam coupled into a UPLC should emerge with the same spot size as that of a beam propagating and diverging in free space over an equivalent physical distance. Simple-cubic photonic crystals can be engineered to self-collimate light. ${ }^{25}$ The ULPCs discussed here are a $20 \times 20 \times 20$ lattice of simplecubic unit cells having a lattice spacing of $a=1.05 \mu \mathrm{m}$ and a fill factor of $\sim 45 \%$, for operation at $\lambda_{0}=1.55 \mu \mathrm{m}$. The UPLCs were fabricated by MPL in IP-Dip photoresist, structurally

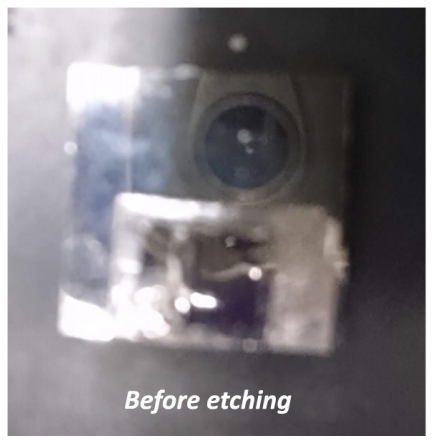

(a)

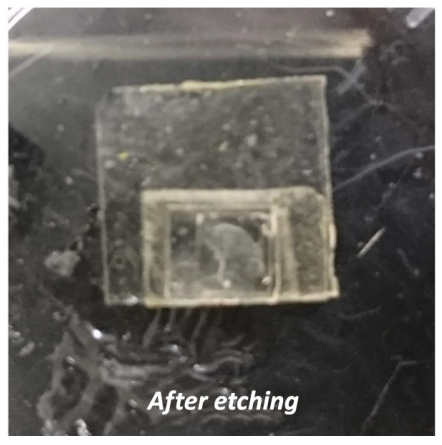

(b)

Fig. 2 Photographs of a sample sputter coated with Au/Pd for SEM imaging (a) before and (b) after etching to remove the metal. After sputter coating with $\mathrm{Au} / \mathrm{Pd}$, the sample is highly reflective. The circular feature visible at the top of the image is the lens of the camera used to record the image, visible as a reflection from the metal-coated substrate. After etching, the sample is highly transmissive, and the reflected image of the camera is no longer visible. 
characterized by sputter coating and SEM imaging, and optical characterized by scannedoptical-fiber measurements.

The difference between metal-coated and etched UPLCs was first examined via visual inspection and optical microscopy. Freshly fabricated microstructures are optically transmissive, but after sputter coating with $\mathrm{Au} / \mathrm{Pd}$, they are opaque and reflective. After etching, the microstructures were restored to optically transmissive, indicating that metal was removed without compromising the structures.

SEM imaging was used to study how etching affects the integrity of the microstructures. SEM images as top and side views of the ULPCs before and after etching are shown in Figs. 3(a) and (b). To obtain the first set of images, samples were fabricated, sputter coated with metal, and imaged by SEM. To obtain the second set of SEM images, the same samples were subjected to the etching process, then sputter coated again for a second round of SEM imaging, to reveal changes potentially caused by etching. The SEM images in Fig. 3 show that structural integrity was maintained throughout the etching process. Individual lines comprising the lattice are rigid and connected to the sample and the form is virtually unchanged. The sample is also free of $I_{2}$ crystals or other deposits, indicating that the rinsing procedure is sufficient to remove the etchant and leave samples free of contaminants. Intriguingly, even the finest defects originating from fabrication are preserved through the etching process. Red ellipses added to the high-magnification images in Figs. 3(b) and 3(d) encircle a defect formed during fabrication of the structure. The defect is clearly visible and unchanged before and after etching. These data confirm that the etching process does not compromise the form or integrity of the microstructures.

The optical behavior of the ULPCs before and after etching was explored using the scannedoptical-fiber system described above, with vertically polarized light having $\lambda_{0}=1.55 \mu \mathrm{m}$. Figure 4 shows the FWHM and transmission efficiency for beams exiting etched and unetched ULPCs. For unetched UPLCs, the beam transmits at an efficiency of $75 \% \pm 3 \%$ with an FWHM of $8 \mu \mathrm{m} \pm 0.3 \mu \mathrm{m}$. The beam size is comparable to that of the input beam, and it transmits with high efficiency, indicating that the device suppresses scatter and beam divergence, consistent with self-collimation. The FWHM and transmission of beams passing through the etched UPLC match those of the unetched device within one standard deviation. These results confirm that etching does not alter the optical function of the nanophotonic device. Even though metal sputtering makes the devices reflective, causing a radical change to their optical properties, the etching process described here restores optical function.

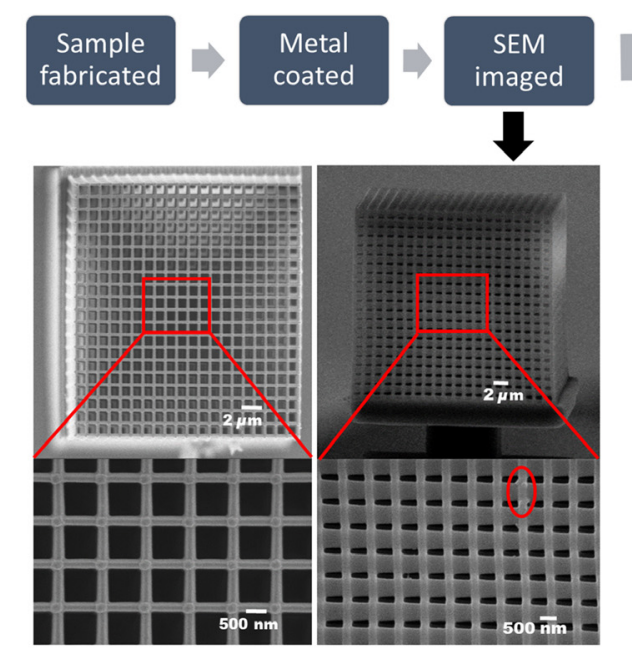

(a)

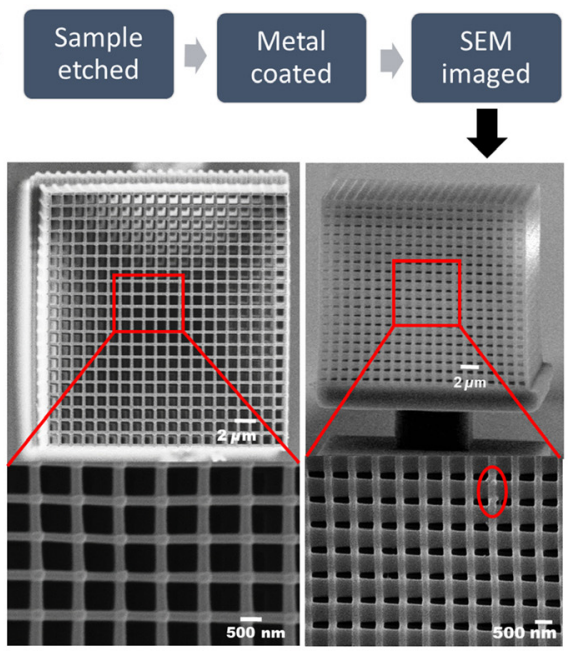

(b)

Fig. 3 SEM images of a UPLC (a) before and (b) after etching. Components $A$ and $C$ are top views, looking down onto the structure and its supporting substrate. Components $B$ and $D$ are side views, looking parallel to the substrate. 


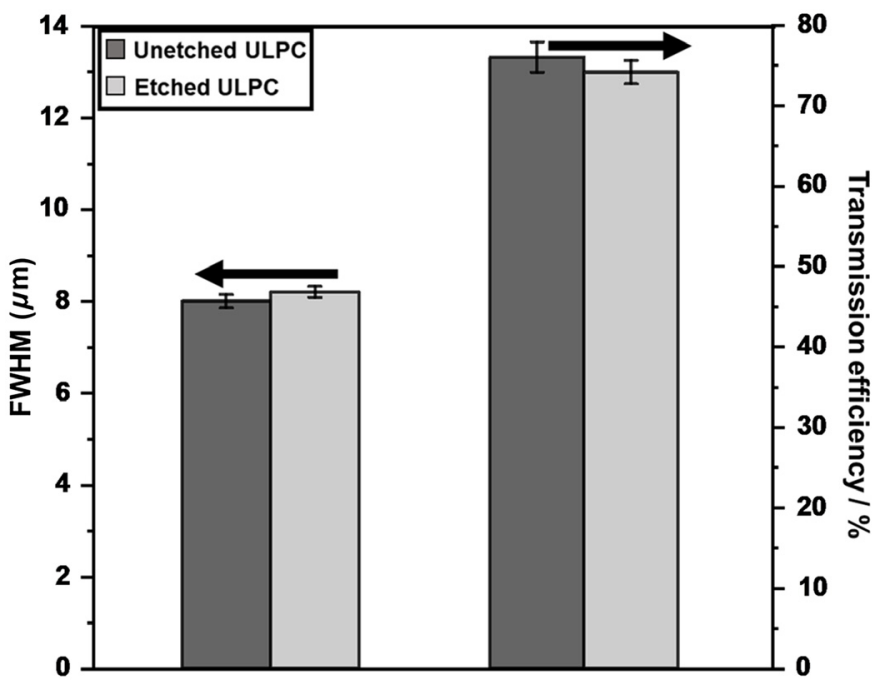

Fig. 4 Comparison of the width and transmission efficiency of a Gaussian beam passing through the UPLC shown in Fig. 3, before and after metal etching.

\subsection{Structural and Optical Characterization of Monolithic Block}

To explore further the effect of sputter coating and etching on the optical behavior of 3D microstructures, a $25 \mu \mathrm{m} \times 25 \mu \mathrm{m} \times 150 \mu \mathrm{m}$ monolithic block was fabricated via MPL in IP-Dip photoresist and then structurally and optically characterized. The monolithic block serves as a complement to the UPLC for assessing the impact of etching. The UPLC is well suited for confirming that the etching process negligibly affects the structure's form; however, it is not straightforward to intuit how the UPLC's optical function would be compromised if metal were still present after etching. In contrast, the simple form of the monolithic block does not reveal subtle changes in structure potentially caused by etching, but it does provide a straightforward means for studying how light transmits through the structure before and after etching.

Figures 5(a) - 5(d) show SEM images of the as fabricated (unetched) monolithic block and the same structure after etching, sputter coating, and reimaging. The structure was optically characterized at $\lambda_{0}=1.55 \mu \mathrm{m}$ (a) immediately after fabrication, (b) after sputter coating with metal
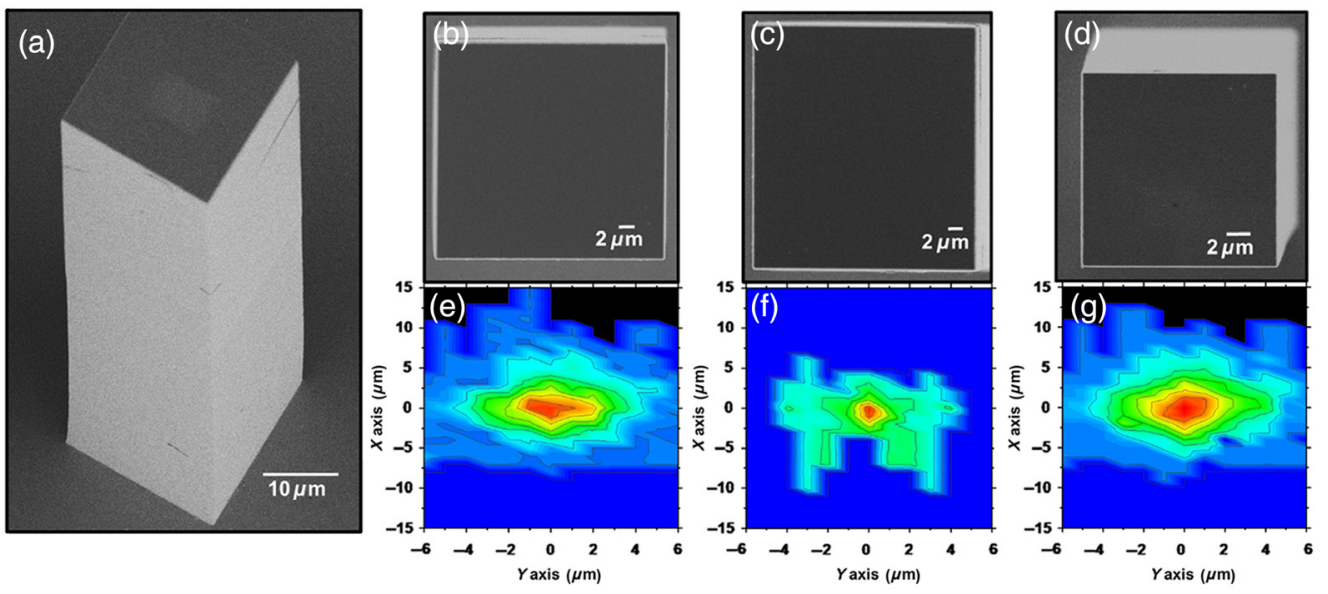

Fig. 5 (a)-(d) SEM images and (e), (f) scanned-fiber optical characterization of the $25 \mu \mathrm{m} \times 25 \mu \mathrm{m} \times 150 \mu \mathrm{m}$ monolithic block at various stages of processing. Intensity profiles measured for an optical beam at $\lambda_{0}=1.55 \mu \mathrm{m}$ exiting from the output face of the block (e) immediately after fabrication, (f) after metal coating, and (g) after etching. (a), (b) Perspective and top views of the block after initial optical characterization shown in (e). (c) A top view of the block after metal sputtering and the second optical characterization shown in (f). (d) A top view of the etched block imaged after the third optical characterization shown in $(\mathrm{g})$. 


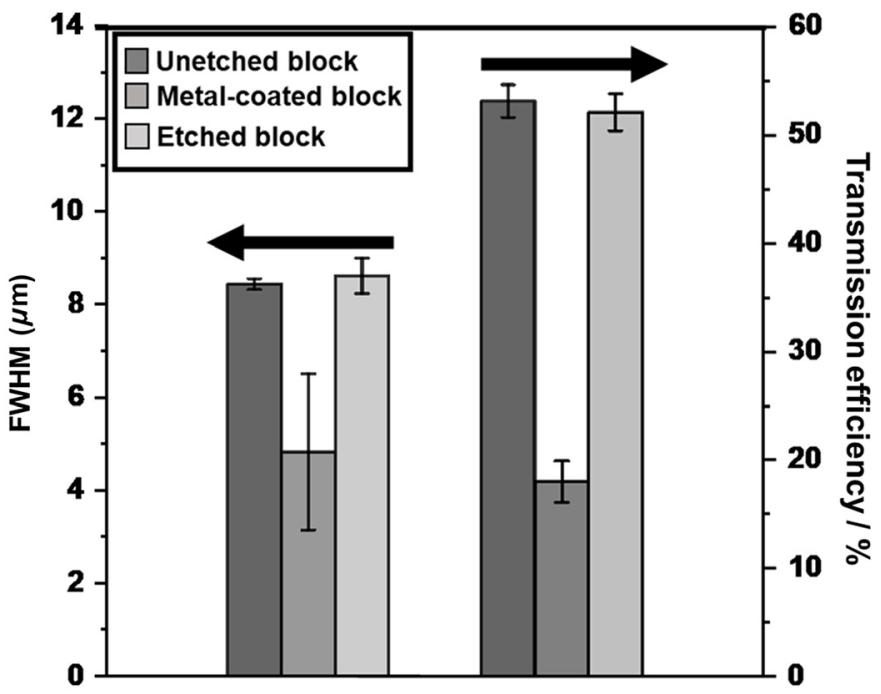

Fig. 6 Comparison of the width and transmission efficiency of a Gaussian beam passing through the monolithic block shown in Fig. 5 immediately after fabrication, after metal coating, and after etching.

for SEM imaging, and (c) again immediately after etching. At all three stages, intensity maps of the transmitted beam were recorded, as shown in Figs. 5(e)-5(g). Line scans through these intensity maps were used to obtain the FWHM of the transmitted beam. The beam widths and optical transmission are presented in Fig. 6.

The transmission efficiency of the as-fabricated block was $53 \% \pm 3 \%$. The material is minimally absorbing at the measurement wavelength, ${ }^{35}$ so the losses are due primarily to reflection at the higher-refractive-index entrance and exit faces and scattering from the surfaces, which have a nanoscale texture due to digital printing by MPL. When the block is sputter coated, the thin metal coating reduces the transmission significantly, down to $18 \% \pm 3 \%$. The profile of the weakly transmitted beam is highly misshapen relative to that observed prior to metal sputtering [compare Fig. 5(e) to Fig. 5(f)], and the FWHM drops to $4 \mu \mathrm{m}$, with high uncertainty due to the beam distortion. Measurements recorded after removing sputtered metal show that the etching process restores the optical transmission $52 \% \pm 3 \%$. The profile of the transmitted beam after etching is also similar to that measured prior to metal deposition, and the FWHM of the corresponding beams are identical within uncertainty. These observations provide additional confirmation that gentle etching using the ethaline-iodine solution can be used to remove sputtered metal and return a microstructure to its original form and optical function.

\section{Conclusion}

A chemical etching process was used to remove metal sputtered onto microstructures for SEM imaging, to restore them to their original form and optical function. The method is based on gentle etching by iodine in ethaline, a eutectic mixture of ethylene glycol and choline chloride. Chloride ions present in the etchant at high-concentration form a coordination complex with noble metal cations, which stabilizes the oxidation product and enables iodine, a comparatively weak oxidizer, to etch the sputter-coated metal. The effects of etching were studied by fabricating 3D microstructures by MPL in a photopolymer and using a scanned-fiber method to characterize their optical function before and after metal coating and etching. SEM imaging before and after a round of etching was used to study how etching affected the structural form. The test structures included a monolithic block, which simply transmits an incident beam and a UPLC, which suppresses divergence of an incident beam by self-collimation as it propagates through the device. The UPLC is a fine-period structure, with deeply submicron-sized features. SEM images of the UPLC recorded before and after etching confirm that the process removes the sputtered noble metal without harming the fragile features of the structure. Even subtle defects in the structure 
that are visible immediately after fabrication are visible after etching. Optical characterization shows that an incident beam travels through the complex lattice without distortion or divergence, both before and after etching. Optical characterization of the monolithic block shows that metal coating severely disrupts transmission of an incident beam, but etching removes the metal and restores transmission and beam shape to that observed prior to metal coating.

Imaging by SEM is widely used to characterize the 2D- and 3D form of micro- and nanoscale structures, including microfluidics, MEMS, micro-optical systems, and MOEMS. Yet SEM is effectively a destructive method insofar as many functions are lost when a given structure is metal coated for high-resolution imaging. The etching method discussed here makes SEM imaging a less destructive technique. The process is demonstrated using a cross-linked acrylate, but it is applicable to structures fabricated in a wide range of non-conducting materials, including other cross-linked polymers, ceramics, glasses, carbides, and composites. Using this etching process in combination with SEM could improve the efficiency of research and device development of microsystems. The etching process could also be useful in industrial and manufacturing settings for quality control and non-destructive testing.

\section{Acknowledgments}

This work was partly supported by the National Science Foundation (Grant No. 1711356).

\section{References}

1. M. Turner et al., "Miniature chiral beamsplitter based on gyroid photonic crystals," Nat. Photonics 7(10), 801-805 (2013).

2. T. Gissibl et al., "Two-photon direct laser writing of ultracompact multi-lens objectives," Nat. Photonics 10, 554-560 (2016).

3. Y. L. Kong et al., "3D Printed quantum dot light-emitting diodes," Nano Lett. 14(12), 7017-7023 (2014).

4. E. Behroodi, H. Latifi, and F. Najafi, "A compact LED-based projection microstereolithography for producing 3D microstructures," Sci. Rep. 9, 19692-19614 (2019).

5. S. V. Murphy and A. Atala, "3D bioprinting of tissues and organs," Nat. Biotechnol. 32(8), 773-785 (2014).

6. S. Zhang and H. Wang, "Current progress in 3D bioprinting of tissue analogs," SLAS Technol. 24(1), 70-78 (2018).

7. Q. Geng et al., "Ultrafast multi-focus 3-D nano-fabrication based on two-photon polymerization," Nat. Commun. 10(1), 2179 (2019).

8. G. Yoon et al., "Fabrication of three-dimensional suspended, interlayered and hierarchical nanostructures by accuracy-improved electron beam lithography overlay," Sci. Rep. 7(1), 6661-6668 (2017).

9. Y. Zhang et al., "User-defined microstructures array fabricated by DMD based multistep lithography with dose modulation," Opt. Express 27, 31956-31966 (2019).

10. R. S. Fischer et al., "Microscopy in 3D: a biologist's toolbox," Trends Cell Biol. 21(12), 682-691 (2011).

11. A. du Plessis et al., "X-ray microcomputed tomography in additive manufacturing: a review of the current technology and applications," 3D Print. Addit. Manuf. 5(3), 227-247 (2018).

12. A. M. Larson, "Multiphoton microscopy," Nat. Photonic 5(1), 1-1 (2011).

13. T. Li et al., "3D printing of hot dog-like biomaterials with hierarchical architecture and distinct bioactivity," Adv. Sci. 6(19), 1901146 (2019).

14. Z. Chen et al., "3D printing of ceramics: a review," J. Eur. Ceram. Soc. 39(4), 661-687 (2019).

15. C. W. Ha, P. Prabhakaran, and Y. Son, "3D-printed polymer/metal hybrid microstructures with ultraprecision for 3D microcoils," 3D Print Addit. Manuf. 6(3), 165-170 (2019).

16. K. Cvecek et al., "Strength of joining seams in glass welded by ultra-fast lasers depending on focus height," J. Laser Micro. Nanoen. 7(1), 68-72 (2012). 
17. E. I. Rau, V. Y. Karaulov, and S. V. Zaitsev, "Backscattered electron detector for 3D microstructure visualization in scanning electron microscopy," Rev. Sci. Instrum. 90(2), 023701 (2019).

18. J. I. Goldstein et al., Eds., "Variable pressure scanning electron microscopy (VPSEM)," in Scanning Electron Microscopy and X-Ray Microanalysis, pp. 173-185, Springer, New York (2017).

19. C. G. Miller et al., "A new laser method for cleaning micropalaeontological specimens," Micropaleontology 23(2), 165-169 (2004).

20. S. Leslie and J. Mitchell, "Removing gold coating from SEM samples," Palaeontology 50(6), 1459-1461 (2007).

21. R. S. Crissman and P. MacCann, "A technique to remove gold-palladium from SEM samples," Micron 10(1), 37-38 (1979).

22. J. Sela and A. Boyde, "Cyanide removal of gold from SEM specimens," J. Microsc. 111(2), 229-231 (1977).

23. H. J. Hansen, "A technique for removing gold from plated calacreous microfossils," Micropaleontology 14(4), 499-500 (1968).

24. D. Jones et al., "Non-destructive, safe removal of conductive metal coatings from fossils: a new solution," Palaeontol. Electron. 15(2), 4T-1-4T-7 (2012).

25. J. L. Digaum et al., "Tight control of light beams in photonic crystals with spatially-variant lattice orientation," Opt. Express 22(21), 25788-25804 (2014).

26. R. Sharma, "Improved system for fabrication and characterization of nanophotonic devices by multi-photon lithography," PhD dissertation, University of Central Florida, Orlando, Florida (2018).

27. A. Ghatak and K. Thyagrajan, Introduction to Fiber Optics, Cambridge University Press (1988).

28. J. Hecht, Understanding Fiber Optics, Prentice-Hall (1999).

29. J. M. Martin, "Coupling efficiency and alignment sensitivity of single mode optical fibers," Master of Science Dissertation, University of Central Florida, Orlando, Florida (1979).

30. A. M. Kowalevicz and F. Bucholtz, "Beam divergence from an SMF-28 optical fiber," Photonics Technology Branch, Optical Sciences Division, Naval Research Laboratory, Washington D.C., pp. 1-13, https://apps.dtic.mil/dtic/tr/fulltext/u2/a456331.pdf (2006).

31. H. Kogelnik, "On the propagation of Gaussian beams of light through lenslike media including those with a loss or gain variation," Appl. Opt. 4, 1562-1569 (1965).

32. R. C. Rumpf et al., "Spatially-variant periodic structures in electromagnetics," Phil. Trans. Royal Soc. A. 373, 20140351-20140359 (2015).

33. V. Bhatt, Essentials of Coordination Chemistry: A Simplified Approach with 3D Visuals, 1st ed., Academic Press, Amsterdam (2015).

34. R. I. C. Etrich and F. Lederer, "Self-collimation of light in three-dimensional photonic crystals," Opt. Express 13(18), 7076-7085 (2005).

35. D. B. Fullager, G. D. Boreman, and T. Hofmann, "Infrared dielectric response of nanoscribe IP-dip and IP-L monomers after polymerization from $250 \mathrm{~cm}^{-1}$ to $6000 \mathrm{~cm}^{-1}$," Opt. Mater. Express 7(3), 888-894 (2017).

Rashi Sharma received her BS degree from Bhim Rao Ambedkar University, Agra, India, and her MS and PhD degrees in chemistry from the University of Central Florida in 2015 and 2018, respectively. She is currently working as a post-doctoral research associate at CREOL of The College of Optics and Photonics at the University of Central Florida. Her research interests include 3D nano- and microfabrication, optoelectronic systems, and infrared transparent material systems.

Jennefir L. Digaum received his BS degree in physics from Mindanao State University and an Erasmus Mundus MS degree in photonics from the University of St. Andrews, Heriot-Watt University, Ghent University, and Vrije University, Brussels, and his $\mathrm{PhD}$ in optics and photonics from CREOL of The College of Optics and Photonics at the University of Central Florida in 2016. He is a principal engineer and RET design lead at Micron Technology supporting development of DRAM, 3D crosspoint, and other emerging memories. 
Hannah West received her BS degree in photonic science and engineering from CREOL of The College of Optics and Photonics at the University of Central Florida. She is currently a master's student and research assistant at the University of Delaware working on RF photonics and fiberoptic communications. She has also investigated 3D micro- and nanofabrication using multiphoton lithography.

Casey M. Schwarz earned her BS, MS, and PhD degrees in physics from the University of Central Florida (UCF) and worked as a post-doctoral research associate investigating processing and properties of innovative materials for photonics. She is an assistant professor of physics at Ursinus College. Her research interests include 3D direct laser writing of multi-component chalcogenide materials, development and design of optical devices, and optical characterization. She is active in STEM outreach and promoting women and under-represented groups in STEM.

Stephen M. Kuebler earned his BS degree in chemistry and his BA degree in German from Tulane University and his DPhil degree in chemistry from the University of Oxford. He is a professor of chemistry and optics at UCF and founding associate director of the UCF Center for Ethics. His group develops new materials and processes for 3D fabrication by multi-photon lithography and uses the technique to fabricate and investigate the innovative 3D nanophotonic devices. He is a senior member of SPIE. 